\title{
Divergence of IL-1, IL-18, and cell death in NLRP3 inflammasomopathies
}

\author{
Susannah D. Brydges, ${ }^{1}$ Lori Broderick, 1,2,3 Matthew D. McGeough,1,2 Carla A. Pena, ${ }^{1,2}$
} James L. Mueller, 1,2,4 and Hal M. Hoffman ${ }^{1,2,3,4}$

1Department of Pediatrics and 2Department of Medicine, UCSD, La Jolla, California, USA. ${ }^{3 R a d y}$ Children's Hospital of San Diego, San Diego, California, USA. ${ }^{4}$ San Diego Branch, Ludwig Institute of Cancer Research, La Jolla, California, USA.

The inflammasome is a cytoplasmic multiprotein complex that promotes proinflammatory cytokine maturation in response to host- and pathogen-derived signals. Missense mutations in cryopyrin (NLRP3) result in a hyperactive inflammasome that drives overproduction of the proinflammatory cytokines IL-1 $\beta$ and IL-18, leading to the cryopyrin-associated periodic syndromes (CAPS) disease spectrum. Mouse lines harboring CAPS-associated mutations in Nlrp3 have elevated levels of IL-1 $\beta$ and IL-18 and closely mimic human disease. To examine the role of inflammasome-driven IL-18 in murine CAPS, we bred Nlrp3 mutations onto an Il18rnull background. Deletion of $I l 18 r$ resulted in partial phenotypic rescue that abolished skin and visceral disease in young mice and normalized serum cytokines to a greater extent than breeding to $I l 1 r$-null mice. Significant systemic inflammation developed in aging Nlrp3 mutant Il18 $r$-null mice, indicating that IL-1 and IL-18 drive pathology at different stages of the disease process. Ongoing inflammation in double-cytokine knockout CAPS mice implicated a role for caspase-1-mediated pyroptosis and confirmed that CAPS is inflammasome dependent. Our results have important implications for patients with CAPS and residual disease, emphasizing the need to explore other NLRP3-mediated pathways and the potential for inflammasome-targeted therapy.

\section{Introduction}

The cryopyrin-associated periodic syndromes (CAPS) are a disease spectrum consisting of three clinically defined autosomal dominant disorders: familial cold autoinflammatory syndrome (FCAS), Muckle-Wells syndrome (MWS), and neonatal-onset multisystem inflammatory disease (NOMID) (1). Missense mutations in cryopyrin (NLRP3) cause the varying degrees of systemic inflammation characteristic of CAPS, including recurrent fever, rash, conjunctivitis, and arthralgia stemming from innate immune dysregulation and overproduction of inflammatory cytokines (2). The NLRP3 protein nucleates the inflammasome, a multiprotein cytoplasmic sensor (3) that responds to varied endogenous and microbe-derived danger signals, including bacterial products, metabolic byproducts such as uric acid (4), and environmental particles such as silica $(5,6)$. Activation of the NLRP3 inflammasome normally requires 2 signals, one to activate transcription of NLRP3, proIL- $1 \beta$, and pro-IL-18 and a second stimulus, resulting in NLRP3 inflammasome formation (3). NLRP3 recruits the adaptor protein pycard (ASC), which then binds multiple molecules of procaspase- 1 , resulting in autocleavage (7). Processed caspase- 1 cleaves both pro-IL-1 $\beta$ and pro-IL-18 to their mature, secreted forms. Biologic therapeutics that target either IL-1 $\beta$ or the IL- 1 receptor (IL-1R) effectively resolve most CAPS symptoms and significantly improve patients' lives (8).

Focusing on IL-1 $\beta$-driven autoinflammation in CAPS, we designed and published a characterization of mouse lines harboring MWS and FCAS mutations in murine Nlrp3 (9). MWS mice

Authorship note: Susannah D. Brydges and Lori Broderick contributed equally to this work.

Conflict of interest: Hal M. Hoffman is a consultant for Regeneron, Novartis, and Sobi and has received research support from GlaxoSmithKline. Susannah D. Brydges is an employee at Regeneron.

Citation for this article: J Clin Invest. 2013;123(11):4695-4705. doi:10.1172/JCI71543. develop neutrophilia in multiple tissues and have elevated serum IL-1 $\beta$ and IL-18. Cultured cells from Nlrp3 mutant mice are hyperresponsive to inflammatory stimuli, similar to patients. Murine CAPS is therefore a reasonable model for human disease. However, despite these similarities, murine CAPS is more severe than its human counterpart, resulting in death in the perinatal (FCAS) or neonatal (MWS) period (9). Abrogating IL-1 signaling either genetically or pharmacologically results in modest improvement of life expectancy in murine CAPS (9) but clearly indicates a role for other mediators in addition to IL-1 $\beta$.

Given the dramatic response to IL-1 inhibition demonstrated by most patients with CAPS, little attention has focused on the effects of IL-18 in the setting of inflammasome-mediated disease. IL-18 is most well-known for its IFN- $\gamma$-inducing ability in the context of IL-12. As such, it is often considered part of the classic Th1 repertoire of mediators, though under certain conditions IL-18 can also drive Th2 and Th17 responses, with both proinflammatory and antiinflammatory results (10). We therefore undertook a thorough examination of the role of IL-18 in CAPS. Hematopoietic cells derived from both mutant mice and monocytes from patients with FCAS hypersecreted IL-18 in response to low amounts of inflammatory stimuli or cold temperature. Breeding Nlrp3 mutations onto an $I l 18 r$-null background resulted in partial phenotypic rescue that abolished skin and visceral disease in young mice. However, significant systemic inflammation developed in aging Nlrp3 mutant Il18rnull mice, indicating that both IL-1 $\beta$ and IL-18 are important for pathogenesis, albeit at different times in the disease process. Additionally, we extended our previous findings that CAPS is inflammasome dependent (9) by demonstrating that intact caspase- 1 is required for disease. Finally, we implicate a role for pyroptosis, a caspase-1-mediated form of cell death. Our results demonstrate the key role of IL-18 in inflammasome-driven disease, yet suggest that other downstream mechanisms besides IL-18-and IL-1 $\beta$-mediated inflammation are involved in this autoinflammatory constellation. 
A

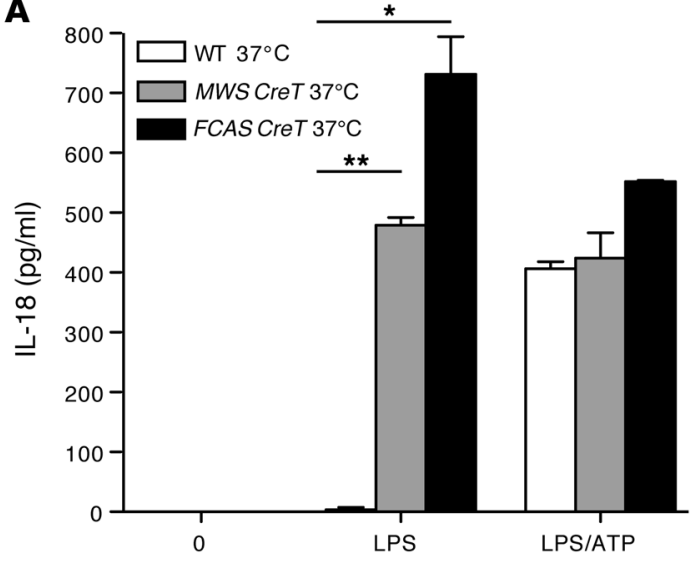

C

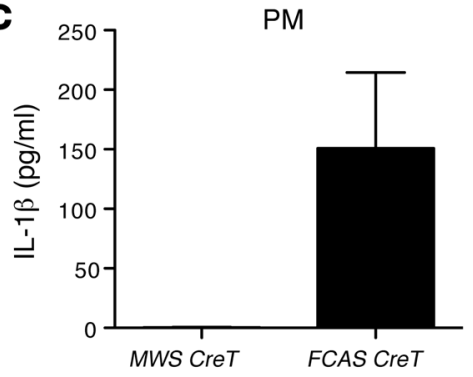

B

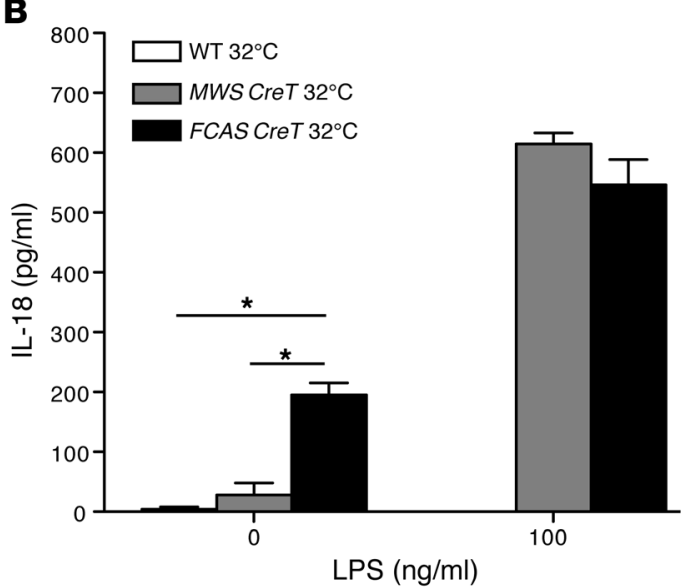

PM

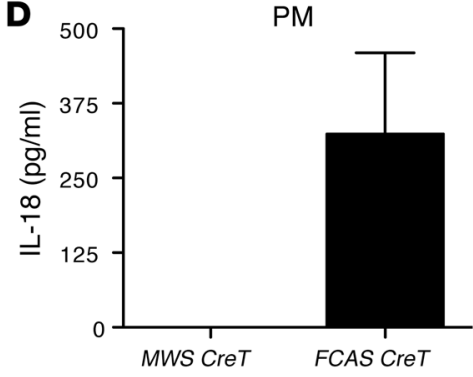

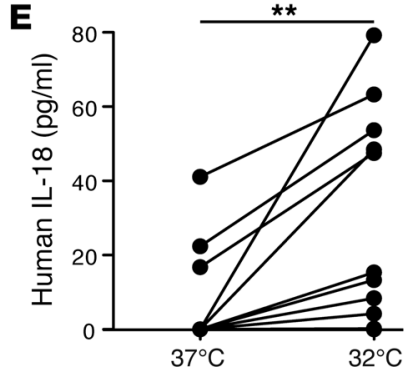

Figure 1

Myeloid cells expressing mutant NLRP3 proteins secrete IL-18. Tamoxifen-treated MWS CreT, FCAS CreT, and littermate WT BMDCs were incubated with pure LPS, with and without ATP at $(\mathbf{A}) 37^{\circ} \mathrm{C}$ or $(\mathbf{B}) 32^{\circ} \mathrm{C}$. IL-18 in the supernatants was measured by ELISA $(n=4$, mean and SEM). Tamoxifen-treated MWS CreT and FCAS CreT peritoneal macrophages (PM) were incubated at $32^{\circ} \mathrm{C}$, and (C) IL- $1 \beta$ and (D) IL-18 were measured in the supernatants by ELISA $\left(n=2\right.$, mean and SEM). (E) Adherent monocytes from patients with FCAS were incubated at $37^{\circ} \mathrm{C}$ or $32^{\circ} \mathrm{C}$, and IL-18 was measured in the culture supernatants by ELISA $(n=10) .{ }^{*} P<0.05,{ }^{* *} P<0.005$, by Student's $t$ test.

\section{Results}

Nlrp3 mutant mouse and CAPS patient hematopoietic cells demonstrate dysregulated IL-18 secretion. While mechanistic and therapeutic studies on patients with autoinflammatory diseases reveal a central role for inflammasome-derived IL- $1 \beta$ in disease pathophysiology, IL-18 has received much less attention. Microbial models of inflammation suggest that the mechanism of inflammasome activation leads differentially to the secretion of IL-1 $\beta$ and IL-18 (11). We have previously introduced inducible Nlrp3 mouse models (MWS CreT and FCAS CreT) that reliably express the human MWS A352V or FCAS L353P alleles (A350V and L351P in mouse NLRP3, respectively) following induction of Cre expression by tamoxifen treatment in culture. Hematopoietic cells from either MWS CreT or FCAS CreT mice secrete high amounts of IL-1 $\beta$ when treated with highly purified LPS, indicating that the mutant NLRP3 inflammasome is constitutively active (9). In contrast, WT cells require both LPS and ATP treatment for inflammasome activation by the classic 2-signal paradigm (12). To determine the signaling requirements for IL-18 secretion in our mutant cells, we performed ELISAs on supernatants from tamoxifen-treated bone marrowderived dendritic cells (BMDCs) from WT, MWS CreT, and FCAS CreT mice. Stimulation with pure LPS alone induced maximal IL-18 release from MWS CreT and FCAS CreT cells, whereas WT BMDCs required both LPS and ATP (Figure 1A). Thus, NLRP3 mutant cells produce both caspase-1-dependent cytokines inde- pendent of the second signal, consistent with the hyperresponsive inflammasome theory of CAPS pathogenesis.

Patients with FCAS have symptomatic flares associated with exposure to cold temperatures (13). Peripheral blood monocytes from patients with FCAS spontaneously secrete IL- $1 \beta$ when incubated at $32^{\circ} \mathrm{C}(14)$, as do murine cells harboring the L351P mutation (9). ELISAs for IL-18 demonstrated that FCAS CreT BMDCs, but not WT or MWS CreT BMDCs, also secrete IL-18 spontaneously when incubated at $32^{\circ} \mathrm{C}$ (Figure 1B). Subsequent treatment with LPS then allowed secretion of IL-18 from MWS CreT cells, emphasizing that incubation at $32^{\circ} \mathrm{C}$ does not significantly impact cell viability. In contrast, WT cells failed to secrete IL-18 at $32^{\circ} \mathrm{C}$, even in the presence of LPS, likely due to the absence of a second signal. Immunoblotting for IL-18 revealed pro-IL-18 in cell lysates at baseline, whereas only mature, cleaved IL-18 was present in stimulated cell culture supernatants, indicating that cells were intact and actively secreting cytokine, rather than simply releasing their cytoplasmic contents (data not shown).

BMDCs must be derived over the span of a week before tamoxifen induction and thus are temporally removed from in vivo bone marrow. To determine whether hypersecretion of IL-18 is a general phenomenon in Nlrp3 mutant mice, we isolated peritoneal macrophages, cells that can be treated ex vivo overnight with tamoxifen to induce NLRP3 expression. Peritoneal cells derived from FCAS CreT mice spontaneously released IL-1 $\beta$ and IL-18 at $32^{\circ} \mathrm{C}$ 
A

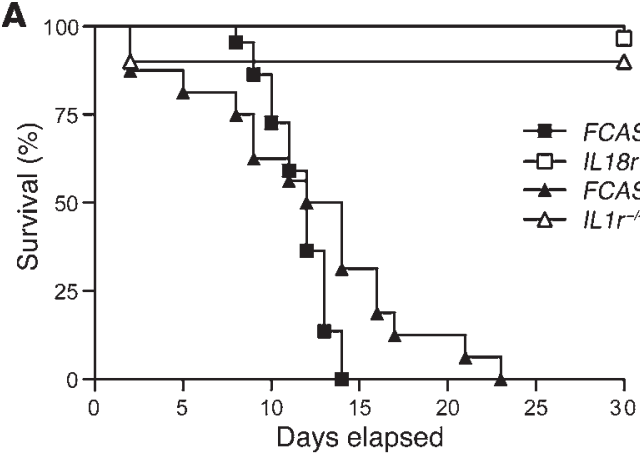

B

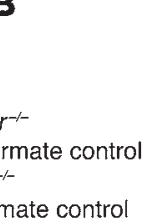

Figure 2

IL-18R deficiency ameliorates inflammation in murine CAPS. (A) Survival and (B) growth of FCAS $1 / 18 r^{-1-}$, $/ 118 r^{-1-}$, FCAS $\| 1 r^{-1-}$, and $/ 11 r^{-1-}$ mice $(n=16-30$ for survival and 1-29 for growth). (C) Survival and (D) growth of MWS, MWS $/ 118 r^{-1-}$, MWS $/ 11 r^{-1-}$, and WT littermates $(n=4-33$ for survival and 1-27 for growth). Statistical significance was tested by log-rank (Mantel-Cox) comparison. Error bars shown for mean daily weights on growth curves are SEM.
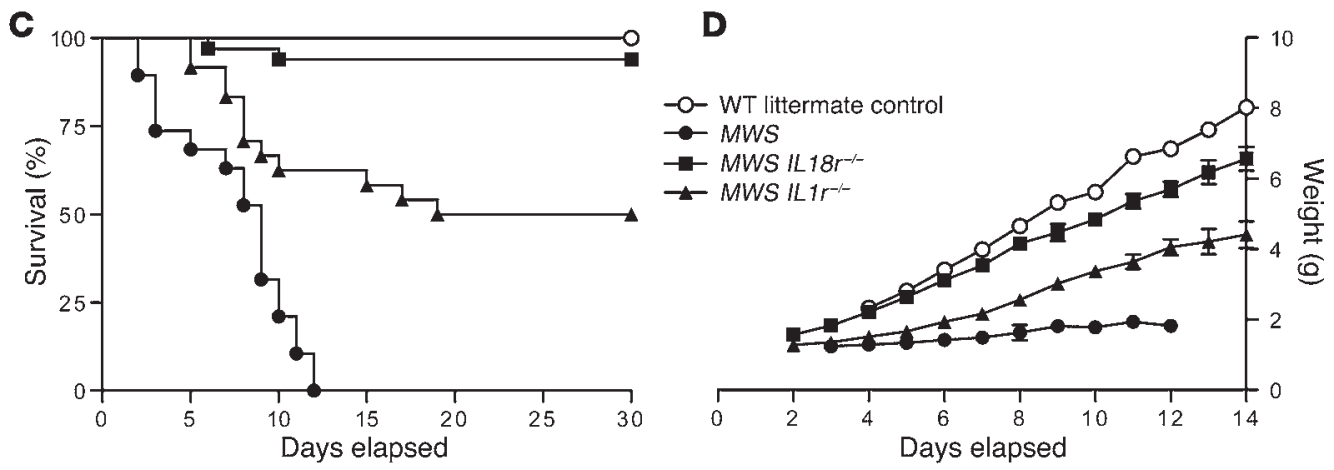

(Figure 1, C and D), whereas MWS CreT peritoneal cells required LPS. Therefore, cold-induced IL-18 secretion is observed in multiple hematopoietic cells types, both fresh and culture-derived, specific to the L351P mutation.

In parallel with our murine studies, we examined the effects of cold on IL-18 secretion from human peripheral blood monocytes. ELISA of culture supernatants demonstrated that peripheral blood monocytes from patients with FCAS carrying the orthologous L353P mutation, as well as other reported FCAS mutations, spontaneously release IL-18 in response to incubation at $32^{\circ} \mathrm{C}$ (Figure 1E). Baseline levels of secreted IL-18 at $37^{\circ} \mathrm{C}$ were variable among patients but not statistically different from normal control human monocytes (data not shown). Taken together, these results imply global dysregulation of the inflammasome in MWS CreT and FCAS CreT mice rather than an IL-1 $\beta$-specific defect, analogous to the human FCAS phenotype (1) and identifying these mice as an appropriate model for the study of IL-18 in CAPS.

Lack of IL-18R signaling ameliorates inflammation in murine CAPS. Most patients with CAPS have a remarkable response to IL-1-targeted therapy, with nearly complete resolution of inflammatory symptoms (8). However, breeding Nlrp3 mutations onto an IL-1R-deficient $\left(\mathrm{IlIr}^{-/}\right)$background resulted in an incomplete phenotypic rescue, with a significant proportion of mixed background (C57BL/ 6 and SV129) FCAS Il1r/- and MWS Il1 $r^{-/}$mice dying within 4 weeks of birth and continued, albeit less severe, skin inflammation in surviving animals (9). Given our findings above that IL-18 dysregulation mirrors that of IL-1 $\beta$, we hypothesized that the residual pathology observed in the absence of IL-1 signaling could be due to IL-18mediated inflammation. We therefore bred FCAS and MWS mutations onto an IL-18 receptor-deficient (IL-18R-deficient; Il18r/-) background to determine the role this inflammasome-dependent cytokine plays during in vivo disease progression.
FCAS mice on an intact cytokine signaling background generally die in utero or in the perinatal period, with obvious skin abscesses and inflammation present (ref. 9 and data not shown), whereas MWS animals live a median of 9 days. In contrast, FCAS Il18r/- animals were born and survived a median of 12 days, similar to FCAS Ill $\mathrm{r}^{-1}$ animals fully backcrossed to C57BL/6 (greater than 10 generations), which occasionally survived a few days longer (Figure 2A). Survival kinetics and growth rates (Figure 2, A and B) are not statistically different between FCAS Ill $r^{--}$and FCAS Il18r/- mice $(P=0.08)$, although the near $100 \%$ survival of FCAS Il18r/- mice to 10 days old suggests a more prominent early role for IL-18. These differences indicate that both inflammasome-dependent cytokines have critical but limited roles in the most severe model of murine CAPS.

To further evaluate the role of IL-18 in early disease development, we studied our milder MWS model on both cytokine receptor knockout backgrounds. Nearly all MWS Il18r/- mice survived to adulthood, with growth curves comparable to those of WT mice, although some pups exhibited slight runting (Figure 2, C and D). MWS Il18r-- mice also lacked the epidermal abscesses, scaling erythema, and alopecia characteristic of MWS animal on an intact cytokine signaling background (data not shown). In contrast, MWS Il1 $r^{-1-}$ mice, fully backcrossed (greater than 10 generations) to C57BL/6 (9), demonstrated delayed growth beginning shortly after birth. Fifty percent of animals then died by 4 weeks of age $(P=0.001)$. Our data suggest that early disease is driven primarily by IL-18, and the absence of IL- 1 signaling provides minimal protection in this initial period. It is possible that the milder disease phenotype characteristic of murine MWS mutation may contribute to the marked difference in pathology observed on the Il18r/- and Il1 $r^{-/}$backgrounds, respectively.

Inflammation in murine CAPS is partially dependent on IL-18 signaling. To elucidate the mechanism behind the marked differences in pro- 


\section{A}
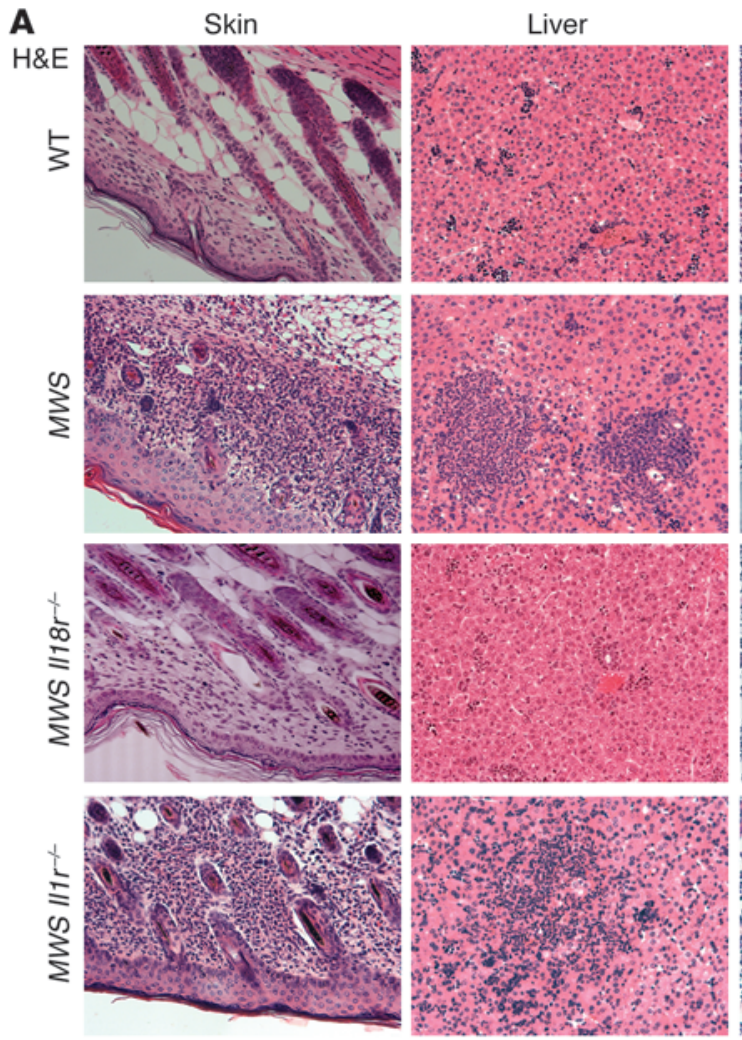

\section{B}
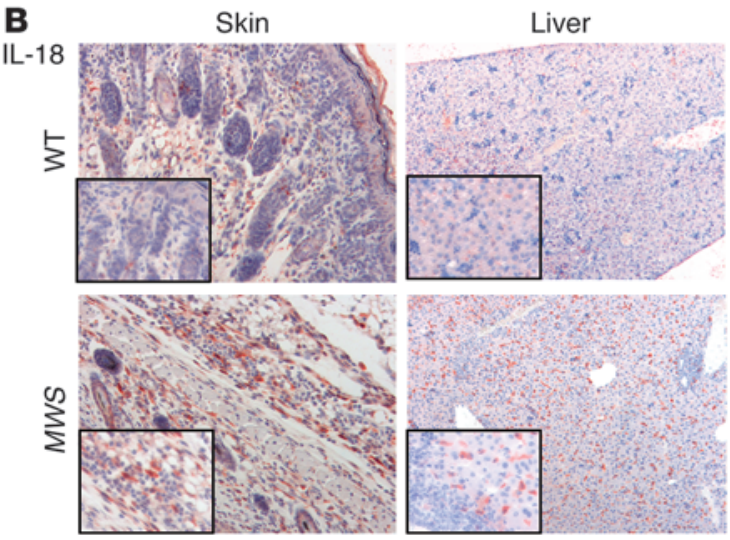

tection provided by the Il18r/- and Il1 $r^{/-}$backgrounds, we undertook a more complete phenotypic analysis of the MWS mice. While MWS Il18r/- pup skin appears grossly similar to that of WT pups, it is still possible that microscopic inflammation exists. We examined H\&E-stained skin sections from 9-day-old WT, MWS, MWS Il1r/-, and MWS Il18r ${ }^{-}$pups and observed numerous dermal granulocytic infiltrations in MWS and MWS Il11 ${ }^{-1}$ skin samples. In contrast, MWS Ill18r/- sections were indistinguishable from those of WT mice, indicating that disruption of IL-18 signaling abrogates superficial and microscopic skin disease (Figure 3A). Similarly, the neutrophilic infiltrates and necrotic regions present in sections of MWS and MWS Il1r $r^{--}$livers were absent in MWS Il18r ${ }^{-1}$ livers (Figure 3A). Cardiac and skeletal muscle as well as pancreatic and intestinal tissues appeared unaffected in all strains (data not shown).

The early mortality of FCAS Il18r-- animals and the slight runting observed in some MWS Il18r/- mice indicate some pathogenic
Bone marrow
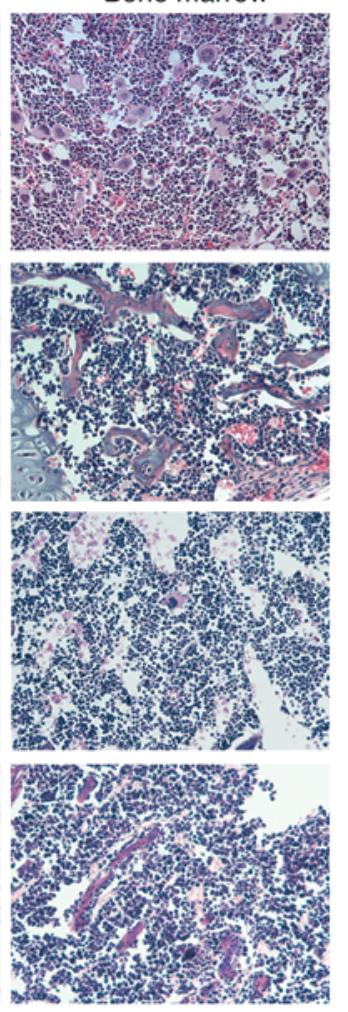

processes must continue unabated on the Il18r/background. H\&E staining of bone marrow revealed the increased presence of polymorphonuclear cells in MWS Il18r-- sections, similar to that in MWS and MWS Il1r ${ }^{-/}$bone marrow (Figure 3A). Thus, while growth, survival, and visceral integrity appear restored in young MWS Il18r/- mice, neutrophilic inflammation continues in the bone marrow. IL-18, like IL- $1 \beta$, is therefore not solely responsible for murine CAPS.

IL-18R signaling drives skin and liver pathology in murine CAPS. The lack of cutaneous inflammation on the $I l 18 r^{-1-}$ background suggests that IL-18 plays an important pathogenic role in the skin. To test whether IL-18 is present in MWS skin, we performed immunohistochemical analyses on sections from 6-day-old WT and MWS mice, when tissue inflammation is fully apparent. IL-18 staining was localized to a thick band beneath the cornified layer in MWS mice and increased in areas of neutrophilic infiltration (Figure 3B). Minimal staining was observed in sections from WT mice. Additionally, stained liver sections from MWS animals demonstrated diffuse IL-18-positive cells throughout, but without noticeable clustering around neutrophilic infiltrates (Figure 3B). Given the dramatic reduction in skin and liver inflammation provided by the $I l 18 r^{/-}$background, it is not surprising that both these tissues contain elevated IL-18 in the intact cytokine signaling state.

IL-18 antibody staining does not distinguish between pro-IL-18 and active, cleaved cytokine; therefore, we examined tissue for evidence of increased IL-18 signaling by performing quantitative RT-PCR analysis on IL-18 signaling targets. IFN- $\gamma$ and IL- 12 transcripts were upregulated (14 and 5 fold, respectively) in skin samples from MWS mice compared with those from WT mice (Supplemental Figure 1; supplemental material available online with this article; doi:10.1172/JCI71543DS1), suggesting that the IL-18 present in the skin is bioactive and further supporting a role for IL-18 in murine CAPS pathogenesis.

Lack of IL-18R signaling normalizes most serum inflammatory protein levels. Serum amyloid A (SAA) levels are an excellent marker for tissue inflammation in patients, and elevation is a prerequisite for amyloid tissue deposits, one of the most severe CAPS sequelae. We previously reported elevated SAA in MWS pups that appeared to be IL-6 dependent (15). As IL-6 production is a common downstream outcome of both IL-1 and IL-18 signaling (16), we examined the effects of cytokine signaling abrogation on SAA levels in 

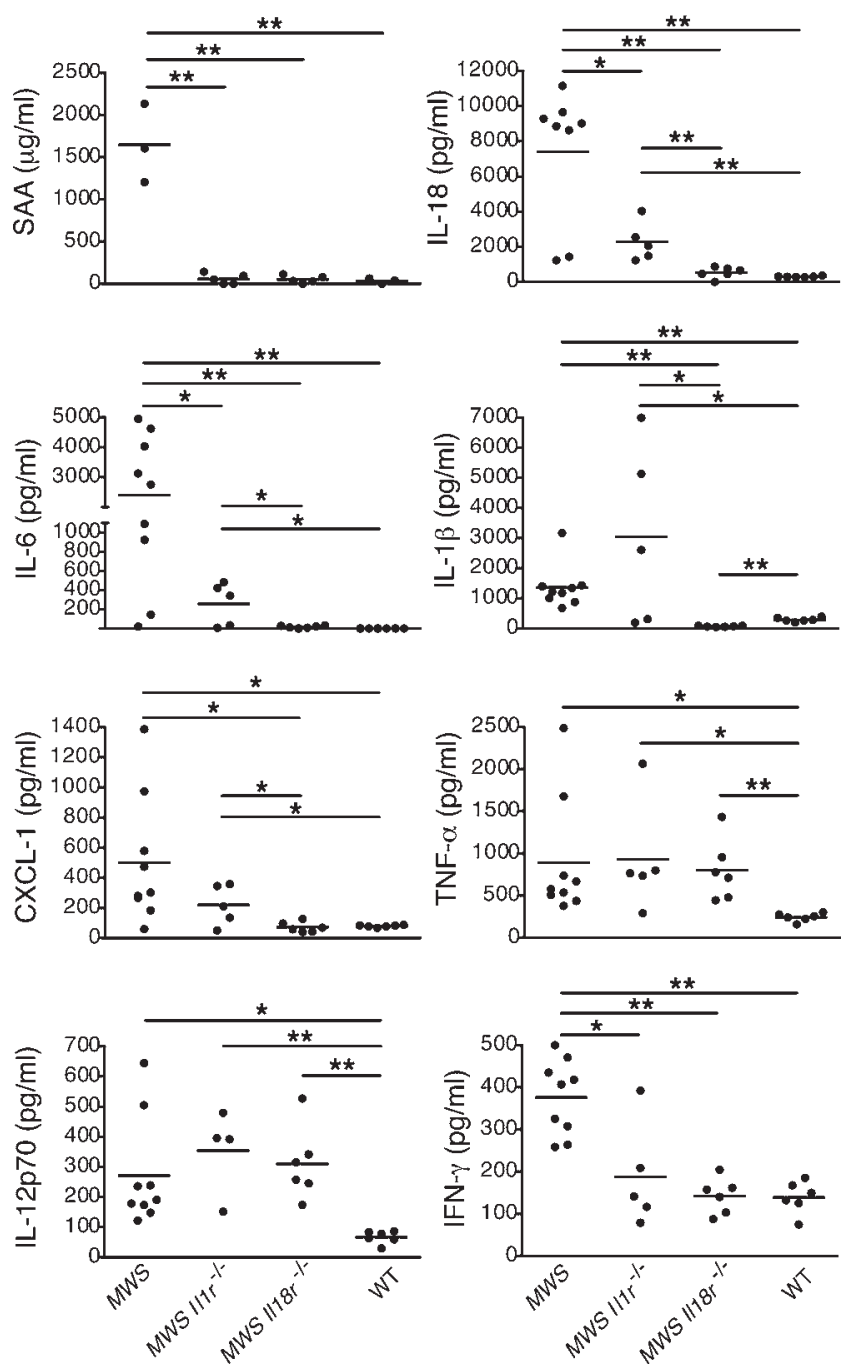

MWS mice. ELISA on serum from MWS, MWS Il18r ${ }^{--}$, MWS Il1r ${ }^{-/}$, and WT mice drawn at days 6-9 of life demonstrated that both knockout backgrounds were similarly effective in reducing SAA to WT levels (Figure 4), despite significant phenotypic differences between MWS Il18r-/ and MWS Il1r ${ }^{-1-}$ mice with respect to survival and tissue inflammation.

In addition to SAA, multiple cytokine mediators were upregulated in MWS serum, particularly factors involved in neutrophil biogenesis and function, such as IL-1 $\beta$, CXCL1 (KC), IL-6, and GCSF (ref. 9 and Figure 4). To determine the systemic effects of ablating IL-18R signaling compared with IL-1, we performed multiplex cytokine analysis on serum from MWS Il18r/- and MWS Il1 $r^{-1-}$ pups as well as MWS and WT mice at days 6-9 after birth. Lack of IL-18R signaling resulted in reduction of IL-18, IL-6, GCSF, eotaxin, and IL-3 to WT levels, whereas the Il1r-1- background reduced but did not completely normalize these mediators (Figure 4 and Supplemental Figure 2A). Levels of Th2 cytokines IL-4, IL,-9 and IL-13 were also lower in serum from MWS Il18r-- pups than MWS Ill $r^{-1-}$ pups (Supplemental Figure 3). Furthermore, levels of IL-1 $\beta$, IL-10, CXCL1, GM-CSF, and MCP-1 were dramatically reduced in MWS Il18r/- pups but not in $\mathrm{MWS} \mathrm{Il1r}^{-/}$pups (Figure 4 and Supplemental Figure 2B). The observation that lack of IL-18R signaling but not lack of IL-1 normalizes both IL-1 $\beta$

\section{Figure 4}

Disruption of IL-18 signaling normalizes key CAPS serum cytokine and SAA levels. Multiplex cytokine and ELISA analysis of serum obtained at days 6-8 from WT, MWS, MWS $/ 11 r^{-1-}$, and MWS $/ 118 r^{-1-}$ pups ( $n \geq 5$ mice); each graph point represents 1 mouse, with mean identified by horizontal bar. ${ }^{\star} P<0.05,{ }^{* *} P<0.005$ by Student's $t$ test.

and IL-18 levels suggests that IL-18 may drive its own production as well as IL-1 $\beta$. Similar to SAA, eliminating either IL-1R or IL-18R signaling effectively decreased IFN- $\gamma$ to WT levels. In contrast, neither IL-18R nor IL-1R signaling affected levels of TNF- $\alpha$, IL-2, IL-12p70, or MIP1 $\alpha$ (Figure 4 and Supplemental Figure 2C). These changes in serum markers further support the phenotypic observations described and the role of IL-18 signaling in early murine CAPS pathogenesis.

Aging MWS Il18r-1- mice develop systemic inflammation. Normalization of nearly all systemic inflammatory mediators and close to $100 \%$ survival to adulthood demonstrated by MWS Il18r/- animals would suggest that abrogating signaling by IL-18 provides a better rescue than preventing signaling via IL-1. However, after 12 weeks of age, certain MWS Il18r-- animals appeared disheveled and thin and occasionally died. This finding is in sharp contrast to our fully backcrossed mice on an Ill $r^{-1}$ background, of which only $25 \%$ survived to adulthood. These few survivors then remained healthy, with no phenotypic indication of ongoing disease, suggesting that if animals can survive this crucial early period, lack of IL-1R signaling can be fully protective. To further investigate temporal differences between the receptor knockout mice and the role of each cytokine in disease development, we extended our studies with an analysis of weight gain in adult mouse strains. At approximately 7 weeks of age, MWS Il18r-/- animals began gaining weight at a slower rate compared with $I l 18 r^{--}$and $I l 1 r^{--}$controls, eventually reaching a plateau at 10 weeks, with little gain thereafter. In contrast, surviving age-matched MWS Il1r-/- mice, runted slightly during the first 6 weeks of life, then followed the control trajectory for the study's duration, eventually catching up to WT counterparts by 16 weeks (Figure 5, A and B).

Throughout all studies, no skin pathology was observed in aging MWS Il18r/- mice; however, peripheral blood and internal examinations revealed ongoing inflammation in these animals. Complete blood counts of MWS $1118 r^{--}$adult mice revealed elevated white blood cell counts, neutrophilia, and thrombocytosis not observed in Il18r-- littermates (Figure 5C). In addition, we observed massive splenomegaly in 15-week-old and older MWS $I l 18 r^{-1}$ mice, with spleen weights ranging from 3 to 6 times those of WT and Il18r/- age-matched controls (Figure 5D). This marked splenomegaly is clearly a late phenomenon in disease progression, as spleens from 8-week-old MWS Il18r/- mice were only slightly larger than those of controls. Spleens isolated from surviving MWS Ill $\mathrm{r}^{-1}$ mice were also slightly enlarged compared with those of WT mice, beginning at 8 weeks, but only by approximately $50 \%$, and, unlike MWS Il18r/- mice, this disparity did not increase with age.

To examine splenic inflammation on a cellular level, we performed flow cytometric analyses on spleens, lymph nodes, and peri-

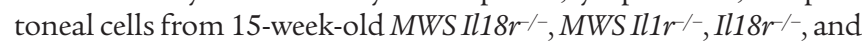
Il1 $r^{-/}$animals. MWS Il18r/- mice demonstrated granular infiltrates of variable intensity in the spleens and lymph nodes, with the most severely affected animals also showing evidence of peritonitis. Tissue infiltrates were predominately CD $11 \mathrm{~b}^{+} / \mathrm{GR} 1^{+}$neutrophils (Sup- 
A

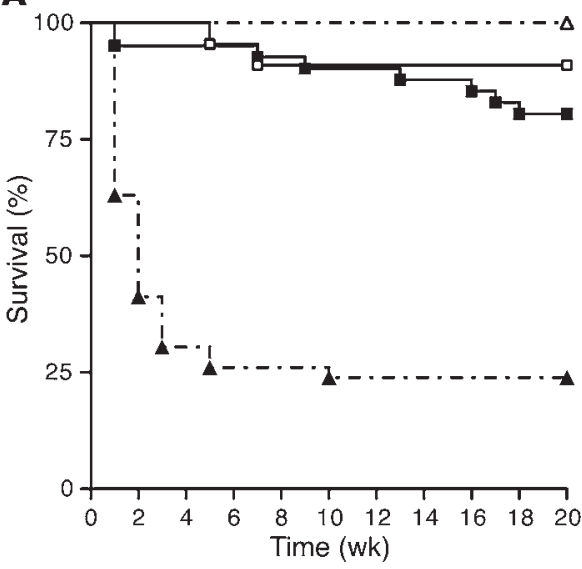

B
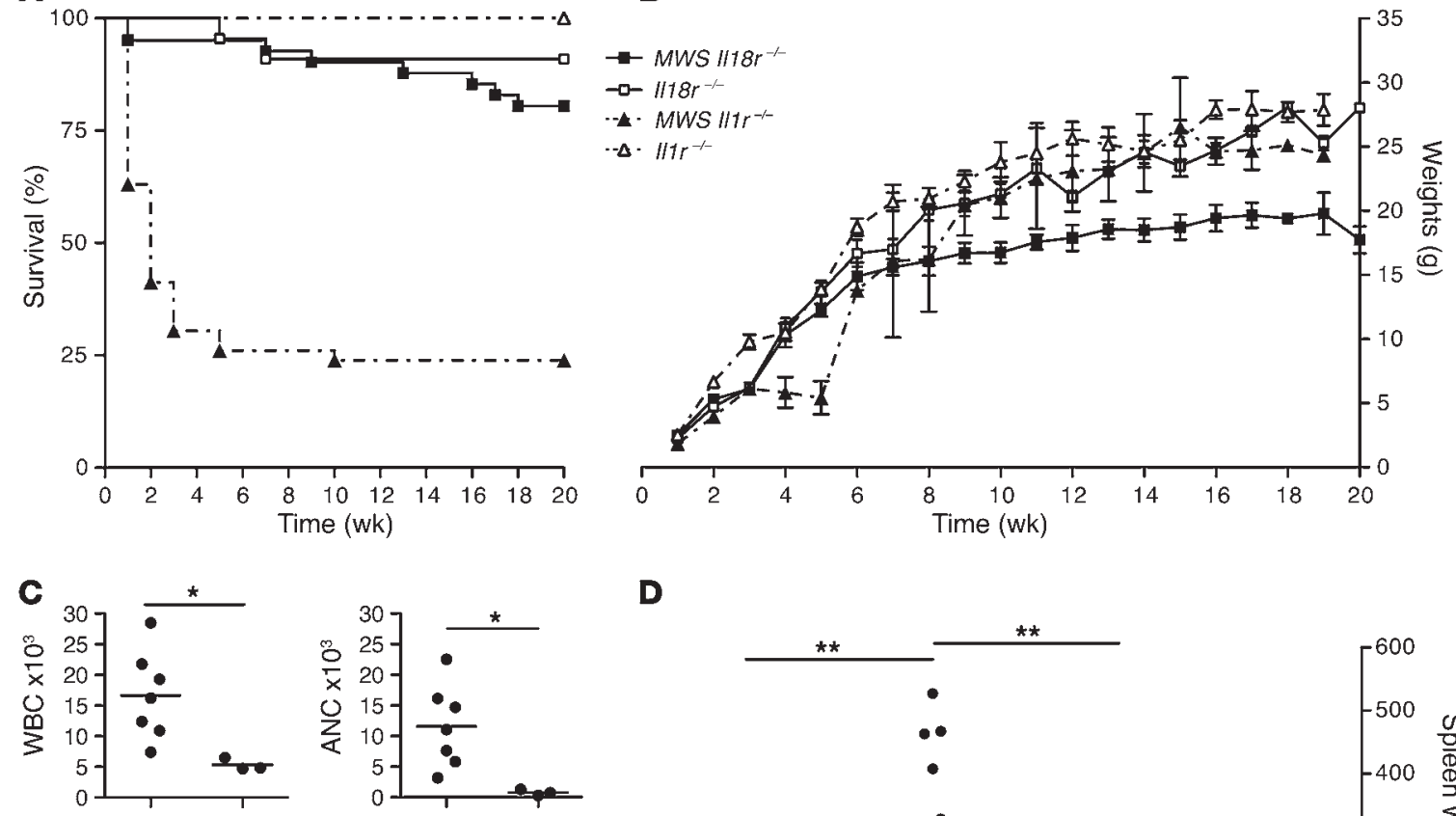

D
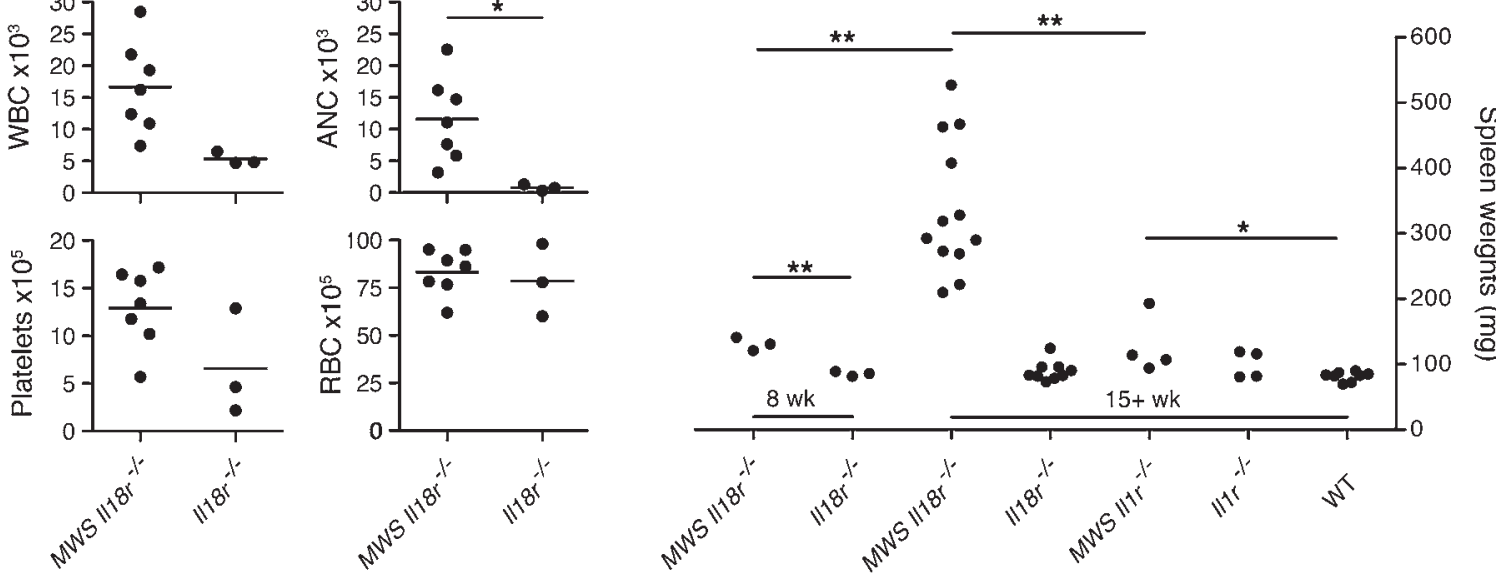

Figure 5

Aging mutant CAPS mice on IL-18R knockout background develop systemic inflammation. (A) Extended survival and (B) growth of $M W S$ I/18 $r^{-1-}$, $\| / 18 r^{-1-}$, MWS $/ / 1 r^{-1-}$, and $/ 11 r^{-1-}$ mice $(n=7-46$ for survival and 1-38 for growth, mean \pm SEM). Statistical significance was tested by log-rank (Mantel-Cox) comparison. (C) Complete peripheral blood counts for MWS $/ / 1 r^{-1-}$ and $I / 18 r^{-1-}$ mice at 16 weeks of age ( $n \geq 3$ mice; each graph point represents 1 mouse, with mean identified by horizontal bar). (D) Spleens from MWS $/ 118 r^{-l_{-}}, \| 18 r^{-1-}$, MWS $/ 11 r^{-1-}$, $/ 111 r^{-1-}$, and WT littermates were weighed at 8 or 15 weeks ( $n=3-12$ mice; each graph point represents 1 mouse, with mean identified by horizontal bar). ${ }^{*}<0.05$, ${ }^{* *} P<0.005$, by Student's $t$ test. WBC, white blood cells; RBC, red blood cells; ANC, absolute neutrophil count.

plemental Figure 4 and data not shown). In contrast, MWS Illr/spleens and lymph nodes did not contain increased CD11b ${ }^{+} / G R 1^{+}$ cells at 15 weeks, and no evidence of peritonitis was observed. Given the persistent bone marrow inflammation observed in younger MWS Il18r/- mice (Figure 3A), and the splenic findings seen later in adults, it is clear that myeloid lineage cells play a pivotal role in continued disease on an $I l 18 r^{-1}$ background.

Somatic cytokine signaling is required for disease pathogenesis in FCAS CreT mice. To examine the relationship of IL-1 and IL-18 in aging mice more specifically, we took advantage of our conditional model, allowing FCAS mice to mature to adulthood prior to disease induction. We treated adult FCAS CreT, FCAS Il1r ${ }^{-1}$ CreT, and FCAS Ill18r ${ }^{-1-}$ CreT mice with tamoxifen to induce the most severe murine CAPS phenotype in vivo. As expected, FCAS CreT mice began to lose weight rapidly (data not shown), and all mice succumbed within 11 days of tamoxifen initiation (Figure 6A). Disruption of IL-18 signaling led to milder pathology, with $50 \%$ of FCAS Il18r/-CreT mice surviving 16 days after tamoxifen initia- tion, consistent with a persistent role for IL-18 in disease. Similar to surviving adult MWS Il1 $\mathrm{r}^{-/}$- mice, disruption of IL-1 signaling resulted in all FCAS Il1r-/- CreT mice surviving beyond 30 days. Taken together, these studies support the importance of both inflammasome cytokines at different disease stages.

We previously showed that expression of mutant Nlrp3 confined to the myeloid cell lineage results in mice with CAPS disease almost indistinguishable from that of mice expressing mutant Nlrp3 universally (9). We took advantage of the rapid disease progression observed in our FCAS-inducible model and performed bone marrow transplant studies, transferring FCAS CreT cells to irradiated WT recipients and vice versa, followed by tamoxifen treatment of engrafted animals. Irradiated WT mice transplanted with FCAS CreT bone marrow lost weight and died within 1 month of tamoxifen induction (Figure 6B), whereas FCAS CreT recipients transplanted with WT bone marrow maintained their weight and survived, with no evidence of disease (Figure 6B). Given our previous findings, the dependence on hematopoietic cells in transfer 


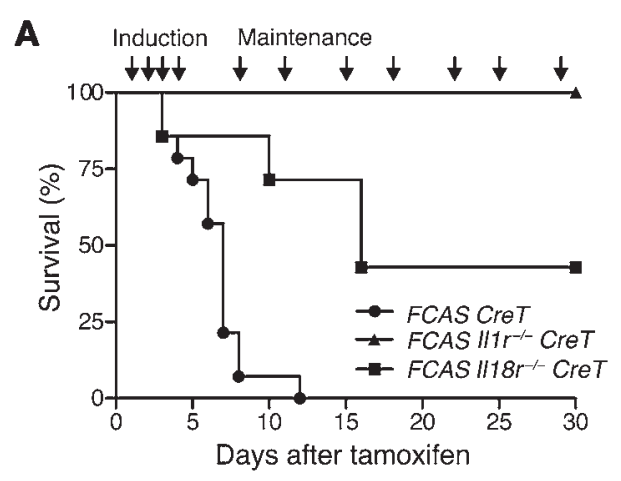

B
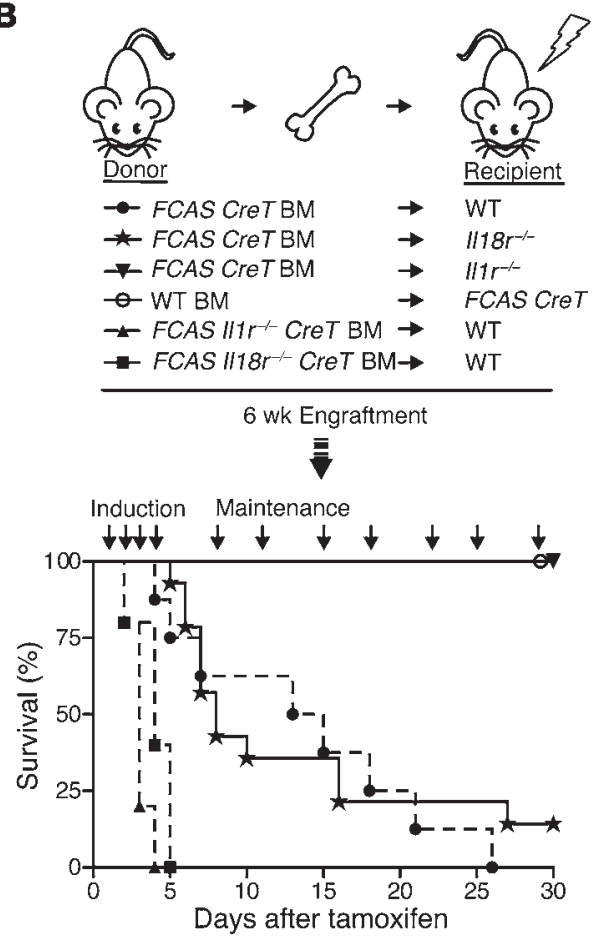

studies is not surprising. The observation that animals expressing mutant Nlrp3 in transplanted hematopoietic cells only (Figure 6B) survive longer than animals expressing the mutation universally (Figure 6A) suggests that the somatic compartment modulates disease and can increase its severity.

To evaluate the role of IL-1 and IL-18 specifically in hematopoietic cells, we performed bone marrow transplant experiments using FCAS Il1r ${ }^{--}$CreT or FCAS Il18r/- CreT mice as donors for WT irradiated recipients. Loss of IL-1R or IL-18R signaling in hematopoietic cells led to full disease in WT recipients and death of all mice by 7 days, a similar and yet slightly more severe time frame than that of WT recipients transplanted with FCAS CreT bone marrow, with IL-1R and IL-18R intact (Figure 6B). Therefore, despite the importance of hematopoietic cells in disease, mechanisms other than IL-1R or IL-18R signaling in the hematopoietic compartment are necessary for pathogenesis, including signaling in the somatic compartment.

The increased survival time of tamoxifen-treated FCAS CreTtransplanted WT mice (Figure 6B) compared with that of nontransplanted, nonirradiated FCAS CreT animals (Figure 6A) prompted us to more extensively examine the role of the somatic

\section{Figure 6}

Inducible and chimeric mutant CAPS models confirm age-related cytokine dependence. (A) Survival of FCAS CreT, FCAS $1 / 18 \mathrm{r}^{-1-}$, or FCAS $/ 11 r^{-1-}$ mice, displayed as days after initiation of tamoxifen treatment ( $n=4-14$ mice). (B) Schematic of bone marrow transplants performed. Survival of transplanted mice, displayed as days after initiation of tamoxifen treatment $(n=5-14$ mice). $P$ values by log-rank (Mantel-Cox) comparison.

compartment, in regards to IL-1R and IL-18R signaling. We performed additional reconstitution experiments transferring FCAS CreT bone marrow into irradiated $I l 1 r^{--}$and $I l 18 r^{-/-}$recipients, followed by tamoxifen treatment, thus exclusively eliminating IL-1R or IL-18R signaling from somatic tissues. The majority of Il18r/- recipients transplanted with FCAS CreT bone marrow died within 2 weeks of treatment initiation; however, a subset ( 2 out of 14 mice) survived for the duration of the experiment. In contrast, $I l 1 r^{/-}$mice were fully protected, with no weight loss or lethality observed (Figure 6B). Thus, IL-1R signaling, and, to a much lesser degree, IL-18R signaling, is necessary in somatic tissues to potentiate disease in adult models of murine CAPS.

Murine CAPS is caspase-1-dependent, but IL-18 and IL-1 $\beta$ are not solely responsible for pathology. Murine CAPS is dependent on an intact inflammasome, as breeding either the FCAS or MWS mutations onto a Pycard ${ }^{-/}$background results in complete phenotypic rescue (9). Since both caspase-1-dependent cytokines, IL-1 $\beta$ and IL-18, contribute to CAPS pathology in mice, yet neither in itself is sufficient for disease, it is possible that abrogating signaling from both cytokines at once will result in a Pycard ${ }^{-/}$-like rescue. We therefore

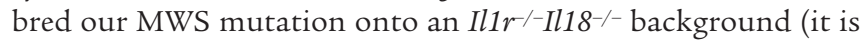

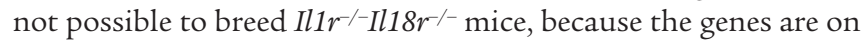
the same chromosome in close proximity). Nearly all MWS Il1 $\mathrm{r}^{\prime-}$ Il18 ${ }^{-1-}$ mice survived to adulthood (Figure 7A) and grew normally (Figure 7B), as expected based on results from MWS Il18r ${ }^{--}$and MWS Il18 $8^{--}$animals (Figure 2, A and B, and Figure 7, A and B). The splenomegaly common to 15-week-old MWS $1118 r^{-/-}$and MWS

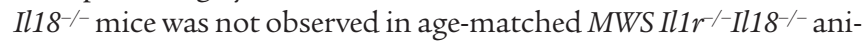
mals (data not shown).

Although the double-null background appears to provide complete resolution of disease resulting from the MWS mutation, a more rigorous test of the efficacy of rescue is the perinatally lethal FCAS mutation. Crossing the FCAS L351P mutation onto the

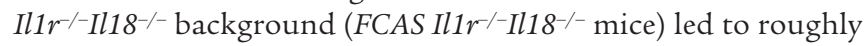
$50 \%$ of animals surviving to adulthood (Figure 7C), with normal weight (Figure 7D) and no obvious skin disease. Although some animals appeared runted early in life, by 12 days of age,

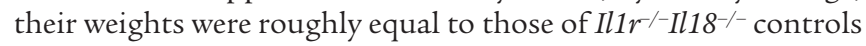

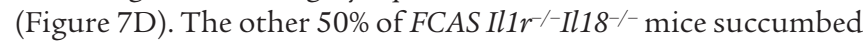
before reaching maturity, with decreasing growth rate and weight loss in certain animals. Therefore, in the context of more severe murine FCAS disease, a double-null background abrogating signaling from both caspase-1-dependent cytokines does improve phenotypic rescue compared with either single-null background alone but fails to eliminate disease completely.

To determine whether the residual, non-IL-1R, non-IL-18driven disease in these mice is caspase- 1 driven or due to caspase-1-independent, inflammasome-dependent effects, we crossed the FCAS allele onto a caspase-1-null background (FCAS Casp $\left.1^{--}\right)$. Resulting mice had normal survival and no evidence of 

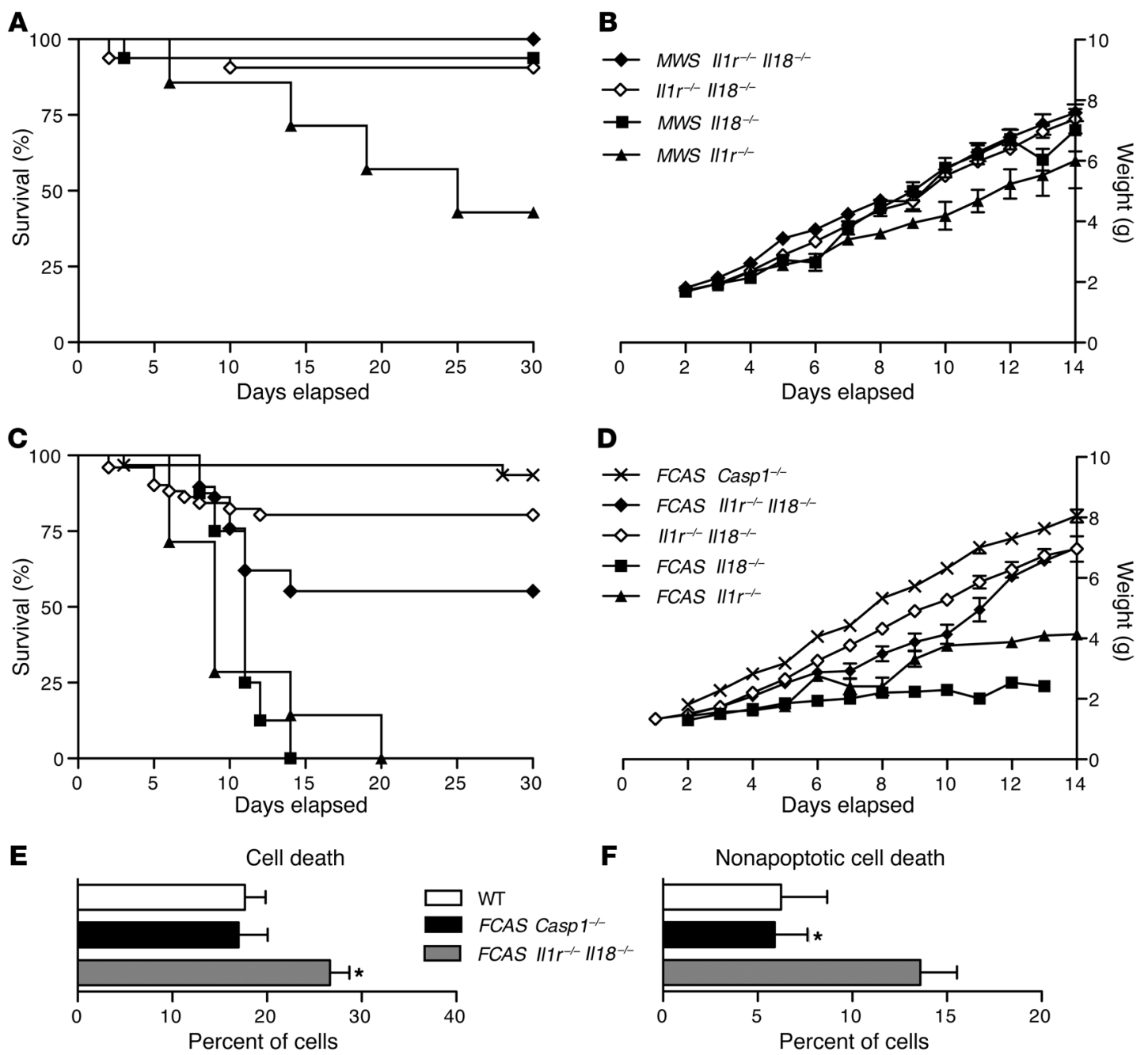

Figure 7

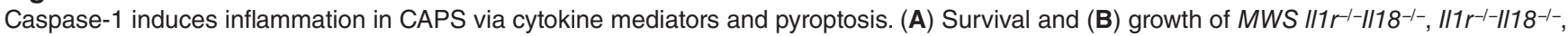

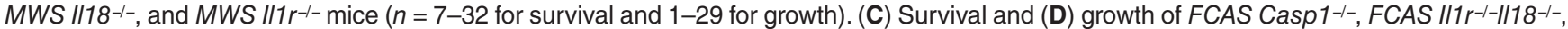
$\left\|1 r^{-1-}\right\| 18^{-1-}$, FCAS $\| 18^{-1-}$, and FCAS $\| 1 r^{-1-}$ mice $(n=7-51$ for survival and 1-40 for growth). Weights are shown as mean \pm SEM. Bone marrow

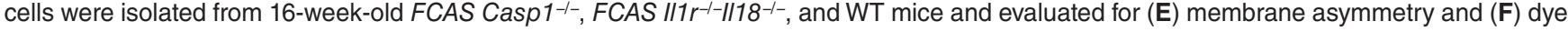
exclusion by flow cytometry. Data are representative of 3 independent experiments (mean \pm SEM shown). ${ }^{*} P<0.05$, by Student's $t$ test.

skin disease, runting, or splenomegaly (Figure 7, C and D). Therefore, murine CAPS is inflammasome and caspase- 1 dependent, but additional mechanisms besides IL-1 $\beta$ and IL-18 must exist.

In addition to cleaving IL-1 $\beta$ and IL-18, caspase- 1 activation also leads to pyroptosis, a proinflammatory cell death pathway (17). Given the role of the NLRP3 inflammasome as a central sensor of cellular stress, it is possible that CAPS mutations and subsequent uncontrolled caspase- 1 activity also lead to enhanced pyroptosis, responsible for the continued inflammation observed in FCAS

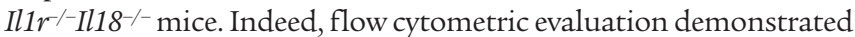
increased cell death in isolated bone marrow-derived cells from FCAS Il1 $r^{-1} I l 1^{-/-}$mice compared with that in WT and FCAS Casp $1^{-1-}$ mice (Figure 7E). This cell death is primarily nonapoptotic in nature, as demonstrated by increased membrane permeability and failure to exclude viability dye (Figure 7F). Treatment with a caspase-1 inhibi-

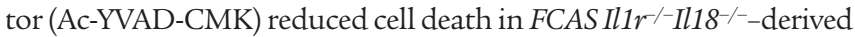
bone marrow cells (data not shown), confirming that pyroptosis was occurring, rather than necrosis or pyronecrosis. Therefore, inflammation in murine CAPS must also be regulated upstream of IL-1 $\beta$ and IL-18, at the level of the NLRP3 inflammasome.

\section{Discussion}

Recent study of the CAPS disease spectrum has led to significant advances in our understanding of the NLRP3 inflammasome and IL-1 $\beta$-mediated inflammation. While translational studies have resulted in vital therapies for patients with CAPS, many questions about the inflammasome and other caspase-1-dependent pathways remain. Inflammasome-mediated IL-18 has largely been overlooked in the context of human disease. In addition, recent studies have demonstrated caspase- 1 and inflammasome functions extending beyond cytokine maturation to cellular death pathways, but whether these processes have any bearing on CAPS remains to be seen. Here, we take advantage of mutant NLRP3 knockin mouse lines to investigate these questions. 
Our studies demonstrate that dysregulated IL-18 secretion occurs from both patient and Nlrp3 mutant mouse cells in a manner similar to IL-1 $\beta$. In vitro, a hallmark of CAPS is lack of reliance on the 2-signal paradigm generally required for secretion of active IL-1 $\beta$. We used this mechanism of inflammasome activation to evaluate IL-18 secretion. Both patient and mouse CAPS cells require some stimulation to transcribe immature cytokine proforms; however, the threshold for mature protein production appears much lower and can be reached with normally inconsequential stimuli such as cold temperature. IL-1 $\beta$ and IL-18 are secreted at similar levels in response to cold by FCAS mutant murine BMDCs, peritoneal macrophages, and FCAS patient peripheral blood monocytes, yet LPS treatment results in nearly 100 -fold excess of IL-1 $\beta$ compared with IL-18. These differences suggest that pathogen-associated molecular patterns and cold are sensed differently by the inflammasome and that IL- $1 \beta$ and IL-18 secretion may be differentially regulated.

In vivo, we demonstrated increased IL-18 in regions of inflamed tissue and increased transcription of some IL-18 targets. Initially, elimination of IL-18R signaling appeared to provide improved phenotypic rescue compared with blockade of IL-1 in young mice, a surprising finding given the profound effect of IL-1 blockade in patients with CAPS. MWS Il18r/- mice are often indistinguishable from their WT littermates at 6 weeks of age. However, upon extended observation, aging MWS $\mathrm{Il}_{18 r^{-1}}$ mice demonstrated evidence of systemic inflammation in the absence of cutaneous pathology sometimes observed in MWS Il1r ${ }^{-1}$ mice. It is therefore likely that the two inflammasome-dependent cytokines have different temporal impacts during the natural progression of disease.

Further dissection of cytokine signaling also revealed spatial differences in the influence of each cytokine. During bone marrow transplant studies, $I_{118 r^{-1}}$ recipients transplanted with FCAS CreT bone marrow succumbed early, whereas $I l 1 r^{/-}$recipients survived, indicating a critical role for IL-1R signaling, but a more limited role for IL-18R, in somatic tissues. Like IL-1 $\beta$, monocytes and dendritic cells are the primary sources of active IL-18, which has a variety of effects. Depending on context, IL-18 stimulates expression of adhesion molecules; induces production of GM-CSF, TNF- $\alpha$, IL-6, and IL- 8 ; enhances neutrophil, NK, and CD8 ${ }^{+} \mathrm{T}$ cell cytotoxicity; and stimulates Th1, Th2, or Th17 responses (18), all hematopoietic targets that would be unaffected in the transplant model. However, unlike IL-1 $\beta$, which requires a stimulus for transcription, pro-IL-18 is constitutively expressed in most tissues by epithelial cells, keratinocytes, and synovial fibroblasts. These steady-state pools of inactive cytokine may then be released under conditions of stress and cleaved exogenously by proteinase-3 (19, 20). Thus, cells that lack all the components for inflammasome formation may still contribute to IL-18-driven inflammation. Therefore, only complete elimination of IL-18R signaling in both the hematopoietic and somatic compartments would lead to a measurable difference in disease activity.

We have extended our previous findings that CAPS is inflammasome dependent (9) by demonstrating that intact caspase-1 is required for disease. Clearly other inflammasome-dependent

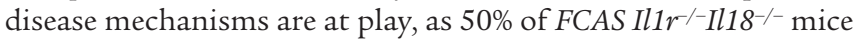
still succumbed to disease before reaching adulthood. It is possi-

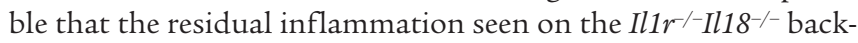
ground is due to increased pyroptosis. In support of this hypoth-

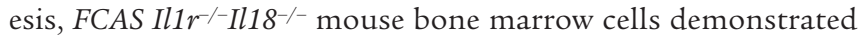
increased caspase-1-dependent, pyroptotic cell death in the bone marrow. Pyroptosis is associated with cleavage and release of IL-18, IL-1 $\beta$, and other inflammatory cellular contents, resulting in activation of nearby cells and potentiating the inflammatory response (21). The relative contribution of this pathway to disease pathogenesis remains to be determined but may be a potential target for therapeutic development.

Our study is limited by the breeding schemes undertaken. By breeding mutant Nlrp3 mice to Ill $r^{--}$and Illsr ${ }^{/-}$mice, we have eliminated specific receptor signaling but cannot account for signaling via alternate receptors or pleiotropic effects due to loss of signaling by alternate ligands. A previous study demonstrated a discrepancy in the susceptibility of $I l 18 r^{-/}$and $I l 18^{-/-}$mice in a model of experimental autoimmune encephalitis, suggesting that other ligand(s) for the IL-18R could exist (22). Therefore, the rescue seen on the $I l 18 r^{-/-}$background could be due to loss of signaling via an unknown ligand independent of IL-18. This scenario is unlikely, since breeding the CAPS mutation onto an $I l 18^{-/-}$ background resulted in an inflammatory phenotype identical to that seen in mutant $I l 18 \mathrm{r}^{-/-}$mice (our unpublished results). In addition, the caspase- 1 knockout mice used in our study were recently found to be deficient in caspase- 11 also. However, studies suggest that caspase- 11 is activated by an inflammasome distinct from NLRP3, making its absence unlikely to significantly influence our results (23).

IL-18 signaling appears to be vital to the initial development of inflammation in murine CAPS. Since only modest improvement is observed when IL-1 signaling is disrupted (9), it is surprising that anti-IL-1 therapies are so effective in patients with CAPS (8). The improvement in inflammation we observed in young mice in the absence of IL-18 signaling suggests that in pups disease is largely driven by IL-18, which then influences IL- $1 \beta$ production. This notion is supported by multiplex results demonstrating that both IL-1 $\beta$ and IL-18 normalize in MWS Il18r-/- serum, while remaining elevated in MWS Il1r ${ }^{-/}$serum. The question therefore remains as to why there are not more anti-IL-1 treatment failures, especially in young patients with CAPS. The differential development of mice in utero compared with human fetuses may provide some insight into this difference. Hematopoietic stem cell development in humans occurs relatively earlier compared with that in mice, at 70 days (first trimester) versus 15.5 days gestation (third trimester) (24), respectively. It is possible that, in humans, the proinflammatory effects of IL-18 occur mainly in utero and IL-1 $\beta$ then drives the pathology observed in young patients with CAPS. To date, neither increased rate of miscarriage, nor extreme prematurity have been described in patients with CAPS. Prematurity has been reported in the related autoinflammatory disease deficiency of the IL-1R antagonist (25), although the role of other cytokines was not investigated.

Despite these questions, our results have important implications for patients with CAPS with residual disease and for patients with conditions involving other NLRP3 and IL- $1 \beta$-mediated pathways $(26,27)$. For patients with other inherited autoinflammatory syndromes (28-35), or more common inflammatory diseases such as heart disease and rheumatoid arthritis, IL-1 blockade has been a successful alternative to traditional therapy, even in patients previously receiving biologics (36-38). However, reports of patients with IL-1 $\beta$-driven diseases that have failed IL-1-targeted therapy are emerging $(39,40)$, suggesting that therapeutics aimed either at IL-18 or upstream at the level of the inflammasome may be valuable. IL-18 binding protein (IL-18BP), a naturally occurring, 
specific inhibitor that blocks the binding of mature IL-18 to its receptor (41), has been used in mouse models of metastatic melanoma and myocardial ischemia (42-44). The elevated expression of IL-18BP in sepsis (45) and macrophage activation syndrome (46) suggests that blockade of IL-18 may be useful in cases refractory to therapy by IL-1 inhibition. Whether similar increases in IL-18BP exist in patients with NLRP3-driven autoinflammatory syndromes remains to be determined.

Recently, small molecule inhibitors have been developed that have the potential for inflammasome-targeted therapy. In vitro experiments with PBMCs from patients with FCAS have demonstrated effectiveness of the orally active ICE/caspase-1 inhibitor, VX-765 (47). Specific caspase-1 or other inflammasome-targeted therapies would affect not only IL- $1 \beta$ and IL-18 secretion but other caspase- 1 substrates and pyroptotic cell death (48). These benefits have been demonstrated in studies of myocardial ischemia, in which IL-1 and IL-18 acted in synergy to cause postischemic dysfunction, which could be prevented, with preservation of cell viability, using caspase 1 inhibitors (49). Investigation into these alternative pathways may be instrumental in our knowledge of the autoinflammatory disease spectrum and function of the NLRP3 inflammasome.

\section{Methods}

Mouse strains. Nlrp $3^{\mathrm{A} 350 \mathrm{VneoR} /+}$ and $N \operatorname{lrp} 3^{\mathrm{L} 351 \mathrm{P} n e o R /+}$ mice were used in the breeding patterns detailed below. These mice are now available through The Jackson Laboratory as B6N.129-Nlrp3 $3^{\text {tm1Hhf}} / J$ and B6N.129-Nlrp3 $3^{\text {tm2Hhf }} / J$ mice, respectively. As previously described, mice were bred to $\mathrm{B} 6 . \mathrm{Cg}-\mathrm{Tg}(\mathrm{Cre})$ Esr1 $)^{5 A m c} / J$ (CreT) mice to generate tamoxifen-inducible mutant NLRP3expressing mice and Nlrp $3^{\mathrm{A} 350 \mathrm{~V} /+} \mathrm{CreT}, \mathrm{Nlrp} 3^{\mathrm{L} 351 \mathrm{P} /+} \mathrm{CreT}$ mice (here referred to as MWS CreT and FCAS CreT mice, respectively) (9). Mutant mice were also bred to B6.129P2-Lyz $2^{\text {tm1(Cre)Ifo } / J ~(C r e L) ~ m i c e ~ t o ~ g e n e r a t e ~ p u p s ~ w i t h ~}$ myeloid lineage restricted expression, $N \operatorname{lrp} 3^{\mathrm{A350V/+}} \mathrm{CreL}$ and $\mathrm{Nlrp} 3^{\mathrm{L} 351 \mathrm{P} /+}$ CreL mice, referred to as MWS and FCAS mice, respectively. B6.129P2Lyz $2^{\operatorname{tm} 1(\mathrm{Cre}) I f o} / J$ (myeloid Cre), B6.Cg-Tg(Cre/Esr1) $5 \mathrm{Amc} / J$ (tamoxifen-inducible Cre ), B6.129S1-IL1r1 $1^{\text {tm IRoml }} / J\left(I l 1 r^{-/}\right)$, B6.129P2-Il18r1 $1^{\text {tm1Aki }} / J\left(I l 18 r^{-/}\right)$, and B6.129P2-Il18 $8^{\text {tm1Aki }} / J\left(I l 18^{-/-}\right)$mice were purchased from The Jackson Laboratory. Casp ${ }^{-/-}$(caspase-1-null) mice were provided by R. Flavell (Howard Hughes Medical Institute, Yale University School of Medicine, New Haven, Connecticut, USA). Littermate controls refer to $N \operatorname{lrp} 3^{\mathrm{A} 350 \mathrm{VneoR} /{ }^{+}}$and $N \operatorname{lrp} 3^{\mathrm{L} 351 \mathrm{PneoR} /{ }^{+}}$mice. A floxed NeoR cassette proximal to the mutated exon prevents expression of the mutant allele. In the absence of Cre expression (as in littermate controls), these mice develop normally, with hemizygous NLRP3 expression stemming from the nondisrupted WT allele. Mice were cared for in accordance with appropriate institutional guidelines.

In vitro cell stimulations. In vitro stimulations for cytokine analysis were performed as previously described (9). Briefly, mouse bone marrow cells were isolated from femurs and tibiae and plated in DMEM supplemented with $10 \%$ FCS, penicillin/streptomycin, L-glutamine, and sodium pyruvate at $4 \times 10^{5}$ cells per well in a 96-well plate with $20 \mathrm{ng} / \mathrm{ml} \mathrm{GM-CSF} \mathrm{(R \& D}$ Systems) for 6 days with 1 media change. Peritoneal macrophages were collected by washing the peritoneal cavity with PBS, followed by plating $4 \times 10^{5}$ cells per well in a 96-well plate overnight. Peritoneal macrophages and BMDCs were then treated overnight with $1 \mu \mathrm{M}$ 4-hydroxy-tamoxifen (Sigma-Aldrich) to stimulate in vitro excision of neoR. Cells were incubated at $37^{\circ} \mathrm{C}$ overnight (crude LPS, Calbiochem) or at $32^{\circ} \mathrm{C}$ or $37^{\circ} \mathrm{C}$ for 4 hours $(100 \mathrm{ng} / \mathrm{ml}$ pure LPS, Alexis Biochemicals Corporation), followed by 30 minutes with $5 \mathrm{mM}$ ATP (Sigma-Aldrich). Supernatants were collected and analyzed for IL- $1 \beta$ or IL-18 by ELISA (R\&D Systems and MBL International). ELISAs were performed in duplicate.
PBMCs were isolated from patients with FCAS (L353P, E525K, Y563N, $\mathrm{M} 659 \mathrm{~K}$ ) and controls by Ficoll gradient and plated at $10^{5}$ cells per well in media without serum in a 96-well plate. Monocytes were allowed to adhere for 3 hours at $37^{\circ} \mathrm{C}$ before the supernatant was replaced with media with $10 \% \mathrm{FCS}$ and incubated overnight at $32^{\circ} \mathrm{C}$ or $37^{\circ} \mathrm{C}$. Secreted IL-18 was measured in cell supernatants by ELISA (MBL International).

Histologic analysis. Mouse skin biopsy samples were fixed in $10 \%$ buffered formalin, embedded in paraffin, sectioned, mounted on slides, deparaffinized, and rehydrated before analysis. Sections were subjected to staining with goat anti-IL-18 (Santa Cruz Biotechnology Inc.) at 1:100 for tissue IL-18 analysis, followed by secondary antibody and hematoxylin counterstaining.

Reverse transcription and quantitative PCR. RNA was isolated from skin biopsy samples from MWS and WT mice using TRIzol (Life Technologies), and cDNA was synthesized using TaqMan Reverse Transcription reagents (Applied Biosystems) per manufacturer's instructions. RT-PCR was performed using TaqMan primer/probe sets for IL-12p35

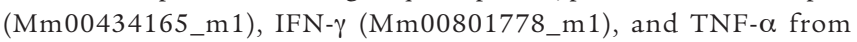
Applied Biosystems. GAPDH amplification was used as a control. Quantitative PCR was performed with a Bio-Rad iCycler using iQ5 software (Supplemental Figure 1).

Flow cytometry. Murine spleens were mechanically dissociated and erythrocytes were removed by hypotonic lysis. $1 \times 10^{6}$ cells were stained with anti-mouse GR-1-APC and anti-mouse CD11b-PE (eBioscience) per manufacturer's directions. For cell death studies, bone marrow cells were harvested from femurs and tibiae by flushing with PBS. Cell membrane asymmetry was evaluated using the Violet Ratiometric Membrane Asymmetry Probe/Dead Cell Apoptosis Kit from Molecular Probes per the manufacturer's instructions. Samples were acquired with a BD Biosciences LSR II cytometer using FACSDiva software.

Bone marrow chimeras. Mice were subjected to lethal total body irradiation $(5.6 \mathrm{~Gy})$ from a ${ }^{137} \mathrm{Cs}$ source, followed by intravenous reconstitution with $5 \times 10^{6}$ bone marrow cells harvested from the femurs of various strains (50). The following transfers were performed: FCAS CreT bone marrow

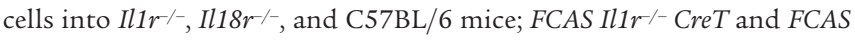
Ill18r/- CreT bone marrow cells into C57BL/6 mice; and C57BL/ 6 cells into FCAS CreT mice, respectively. Mice were allowed to engraft for 6 weeks and given trimethoprim-sulfamethoxazole (Hi-Tech Pharmacal) in the water ad libitum. To induce mutant NLRP3 expression, mice were injected intraperitoneally with $50 \mathrm{mg} / \mathrm{kg}$ tamoxifen free base stock (MP Biomedicals) in 9:1 sunflower oil (Helianthus annus [Sigma-Aldrich])/ethanol daily for 4 days and then twice weekly for the remainder of the experiment. Mice were monitored and weighed daily.

Peripheral blood analysis. Peripheral blood from 6- to 9-day-old pups was obtained following decapitation. Serum was analyzed by ELISA (SAA, Tridelta Development) or cytokine multiplex immunoassay (BioPlex Mouse Cytokine Assays, Bio-Rad) per manufacturer's instructions. Peripheral blood was collected from adult mice by submandibular venous puncture, and complete blood counts were performed by the UCSD ACP Diagnostic Laboratory.

Statistics. Statistical analyses and graphing were performed in Microsoft Excel and GraphPad Prism programs, with the 2-tailed Student's $t$ test and log-rank (Mantel-Cox) test. Flow cytometry data were analyzed by FlowJo software. A $P$ value less than 0.05 was considered statistically significant.

Study approval. Human subjects provided written informed consent, prior to inclusion, under protocols approved by the UCSD Human Research Protections Program and were performed in accordance with the 1964 Declaration of Helsinki and its later amendments. Murine experimental protocols were approved by the UCSD Institutional Animal Care and Use Committee. 


\section{Acknowledgments}

The authors would like to thank Debbie Chen, Akemi Brown, and Sophie Jang for technical support. This work was supported by NIH grants R01AI52430 (to H.M. Hoffman) and T32AI007469 (to L. Broderick), the Arthritis National Research Foundation (to S.D. Brydges), and GlaxoSmithKline (to H.M. Hoffman).

Received for publication June 17, 2013, and accepted in revised form August 1, 2013.

1. Aksentijevich I, et al. The clinical continuum of cryopyrinopathies: novel CIAS1 mutations in North American patients and a new cryopyrin model. Arthritis Rheum. 2007;56(4):1273-1285

2. Hoffman HM, Mueller JL, Broide DH, Wanderer AA, Kolodner RD. Mutation of a new gene encoding a putative pyrin-like protein causes familial cold autoinflammatory syndrome and Muckle-Wells syndrome. Nat Genet. 2001;29(3):301-305.

3. Martinon F, Burns K, Tschopp J. The inflammasome: a molecular platform triggering activation of inflammatory caspases and processing of proIL-beta. Mol Cell. 2002;10(2):417-426.

4. Martinon F, Petrilli V, Mayor A, Tardivel A, Tschopp J. Gout-associated uric acid crystals activate the NALP3 inflammasome. Nature. 2006;440(7081):237-241.

5. Cassel SL, et al. The Nalp3 inflammasome is essential for the development of silicosis. Proc Natl Acad Sci US A. 2008;105(26):9035-9040.

6. Dostert C, Petrilli V, Van Bruggen R, Steele C, Mossman BT, Tschopp J. Innate immune activation through Nalp3 inflammasome sensing of asbestos and silica. Science. 2008;320(5876):674-677.

7. Srinivasula SM, Poyet JL, Razmara M, Datta P, Zhang Z, Alnemri ES. The PYRIN-CARD protein ASC is an activating adaptor for caspase-1. J Biol Chem. 2002;277(24):21119-21122.

8. Doherty TA, Brydges SD, Hoffman HM. Autoinflammation: translating mechanism to therapy. J Leukoc Biol. 2011;90(1):37-47.

9. Brydges SD, et al. Inflammasome-mediated disease animal models reveal roles for innate but not adaptive immunity. Immunity. 2009;30(6):875-887.

10. Smith DE. The biological paths of IL-1 family members IL-18 and IL-33. J Leukoc Biol. 2011; 89(3):383-392.

11. Schmidt RL, Lenz LL. Distinct licensing of IL-18 and IL-1beta secretion in response to NLRP3 inflammasome activation. PLoS One. 2012;7(9):e45186.

12. Bauernfeind FG, et al. Cutting edge: NF-kappaB activating pattern recognition and cytokine receptors license NLRP3 inflammasome activation by regulating NLRP3 expression. J Immunol. 2009; 183(2):787-791.

13. Hoffman HM, Wanderer AA, Broide DH. Familial cold autoinflammatory syndrome: phenotype and genotype of an autosomal dominant periodic fever. J Allergy Clin Immunol. 2001;108(4):615-620.

14. Rosengren $S$, et al. Monocytes from familial cold autoinflammatory syndrome patients are activated by mild hypothermia. J Allergy Clin Immunol. 2007; 119(4):991-996

15. McGeough MD, et al. Cutting edge: IL- 6 is a marker of inflammation with no direct role in inflammasome-mediated mouse models. J Immunol. 2012; 189(6):2707-2711.

16. Lee JK, Kim SH, Lewis EC, Azam T, Reznikov LL, Dinarello CA. Differences in signaling pathways by IL-1beta and IL-18. Proc Natl Acad Sci U S A. 2004; 101(23):8815-8820.

17. Fink SL, Cookson BT. Apoptosis, pyroptosis, and necrosis: mechanistic description of dead and dying eukaryotic cells. Infect Immun. 2005; 73(4):1907-1916.

Address correspondence to: Hal Hoffman, 9500 Gilman Drive, Mail code 0635, La Jolla, California 92093-0635, USA. Phone: 858.534.2108; Fax: 858.534.2110; E-mail: hahoffman@ucsd.edu.

Susannah D. Brydges's present address is: Regeneron Pharmaceuticals Inc., Tarrytown, New York, USA.

James L. Mueller's present address is: UCSF, San Francisco, California, USA.

18. Arend WP, Palmer G, Gabay C. IL-1, IL-18, and IL-33 families of cytokines. Immunol Rev. 2008;223:20-38.

19. Dinarello CA, Fantuzzi G. Interleukin-18 and host defense against infection. J Infect Dis. 2003; 187(suppl 2):S370-S384.

20. Sugawara S, et al. Neutrophil proteinase 3-mediated induction of bioactive IL-18 secretion by human oral epithelial cells. J Immunol. 2001;167(11):6568-6575.

21. Bergsbaken T, Fink SL, den Hartigh AB, Loomis WP, Cookson BT. Coordinated host responses during pyroptosis: caspase-1-dependent lysosome exocytosis and inflammatory cytokine maturation. J Immunol. 2011;187(5):2748-2754.

22. Gutcher I, Urich E, Wolter K, Prinz M, Becher B. Interleukin 18-independent engagement of interleukin 18 receptor-alpha is required for autoimmune inflammation. Nat Immunol. 2006;7(9):946-953.

23. Kayagaki N, et al. Non-canonical inflammasome activation targets caspase-11. Nature. 2011; 479(7371):117-121.

24. Mikkola HK, Orkin SH. The journey of developing hematopoietic stem cells. Development. 2006; 133(19):3733-3744.

25. Aliok E, et al. A novel mutation in the interleukin-1 receptor antagonist associated with intrauterine disease onset. Clin Immunol. 2012;145(1):77-81.

26. Dinarello CA. Anti-inflammatory agents: present and future. Cell. 2010;140(6):935-950.

27. Goldbach-Mansky R. Immunology in clinic review series; focus on autoinflammatory diseases: update on monogenic autoinflammatory diseases: the role of interleukin (IL)-1 and an emerging role for cytokines beyond IL-1. Clin Exp Immunol. 2012; 167(3):391-404.

28. Meinzer U, Quartier P, Alexandra JF, Hentgen V, Retornaz F, Kone-Paut I. Interleukin-1 targeting drugs in familial Mediterranean fever: a case series and a review of the literature. Semin Arthritis Rheum. 2011;41(2):265-271.

29. Mitroulis I, Papadopoulos VP, Konstantinidis T, Ritis K. Anakinra suppresses familial Mediterranean fever crises in a colchicine-resistant patient. Neth J Med. 2008;66(11):489-491.

30. Mitroulis I, Skendros P, Oikonomou A, Tzioufas AG, Ritis K. The efficacy of canakinumab in the treatment of a patient with familial Mediterranean fever and longstanding destructive arthritis. Ann Rheum Dis. 2011;70(7):1347-1348.

31. Ozen S, Bilginer Y, Aktay Ayaz N, Calguneri M. Anti-interleukin 1 treatment for patients with familial Mediterranean fever resistant to colchicine. J Rheumatol. 2011;38(3):516-518.

32. Cailliez M, et al. Anakinra is safe and effective in controlling hyperimmunoglobulinaemia D syndrome-associated febrile crisis. J Inherit Metab Dis. 2006;29(6):763

33. Lequerre $T$, et al. Interleukin-1 receptor antagonist (anakinra) treatment in patients with systemiconset juvenile idiopathic arthritis or adult onset Still disease: preliminary experience in France. Ann Rheum Dis. 2008;67(3):302-308.

34. Gattorno M, et al. Persistent efficacy of anakinra in patients with tumor necrosis factor receptor-associated periodic syndrome. Arthritis Rheum. 2008;
58(5):1516-1520.

35. Obici L, et al. Favourable and sustained response to anakinra in tumour necrosis factor receptor-associated periodic syndrome (TRAPS) with or without AA amyloidosis. Ann Rheum Dis. 2011;70(8):1511-1512.

36. Abbate A, et al. Interleukin-1 blockade with anakinra to prevent adverse cardiac remodeling after acute myocardial infarction (Virginia Commonwealth University Anakinra Remodeling Trial [VCU-ART] Pilot study). Am J Cardiol. 2010;105(10):1371-1377.e1.

37. Fleischmann R, Stern R, Iqbal I. Anakinra: an inhibitor of IL-1 for the treatment of rheumatoid arthritis. Expert Opin Biol Ther. 2004;4(8):1333-1344.

38. McGonagle D, Tan AL, Shankaranarayana S, Madden J, Emery P, McDermott MF. Management of treatment resistant inflammation of acute on chronic tophaceous gout with anakinra. Ann Rheum Dis. 2007;66(12):1683-1684.

39. Buch MH, et al. Lack of response to anakinra in rheumatoid arthritis following failure of tumor necrosis factor alpha blockade. Arthritis Rheum. 2004; 50(3):725-728.

40. Lin Z, et al. Failure of anakinra treatment of pyoderma gangrenosum in an IBD patient and relevance to the PSTPIP1 gene. Inflamm Bowel Dis. 2011; 17(6):E41-E42.

41. Novick D, Kim SH, Fantuzzi G. Interleukin-18 binding protein: a novel modulator of the Th1 cytokine response. Immunity. 1999;10(1):127-136.

42. Raeburn CD, et al. Neutralization of IL-18 attenuates lipopolysaccharide-induced myocardial dysfunction. Am J Physiol Heart Circ Physiol. 2002; 283(2):H650-H657.

43. Vidal-Vanaclocha R, et al. IL-18 regulates IL-1betadependent hepatic melanoma metastasis via vascular cell adhesion molecule-1. Proc Natl Acad Sci US A. 2000;97(2):734-739.

44. Carrascal MT, et al. Interleukin-18 binding protein reduces b16 melanoma hepatic metastasis by neutralizing adhesiveness and growth factors of sinusoidal endothelium. Cancer Res. 2003;63(2):491-497.

45. Novick D, et al. A novel IL-18BP ELISA shows elevated serum IL-18BP in sepsis and extensive decrease of free IL-18. Cytokine. 2001;14(6):334-342.

46. Mazodier K, et al. Severe imbalance of IL-18/ IL-18BP in patients with secondary hemophagocytic syndrome. Blood. 2005;106(10):3483-3489.

47. Stack JH, et al. IL-converting enzyme/caspase-1 inhibitor VX-765 blocks the hypersensitive response to an inflammatory stimulus in monocytes from familial cold autoinflammatory syndrome patients. JImmunol. 2005;175(4):2630-2634.

48. Denes A, Lopez-Castejon G, Brough D. Caspase-1: is IL-1 just the tip of the ICEberg? Cell Death Dis. 2012;3:e338.

49. Pomerantz BJ, Reznikov LL, Harken AH, Dinarello CA. Inhibition of caspase 1 reduces human myocardia ischemic dysfunction via inhibition of IL-18 and IL-1beta. Proc Natl Acad Sci U S A. 2001; 98(5):2871-2876.

50. Shigeoka AA, et al. An inflammasome-independent role for epithelial-expressed Nlrp3 in renal ischemia-reperfusion injury. J Immunol. 2010; 185(10):6277-6285 